\title{
Protée
}

\section{L’altérité originaire ou les mirages fondateurs de l'identité}

\section{Michel Bernard}

Volume 29, numéro 2, 2001

Danse et altérité

URI : https://id.erudit.org/iderudit/030622ar

DOI : https://doi.org/10.7202/030622ar

Aller au sommaire du numéro

Éditeur(s)

Département des arts et lettres - Université du Québec à Chicoutimi

ISSN

0300-3523 (imprimé)

1708-2307 (numérique)

Découvrir la revue

Citer cet article

Bernard, M. (2001). L'altérité originaire ou les mirages fondateurs de l'identité. Protée, 29(2), 7-24. https://doi.org/10.7202/030622ar

\section{Résumé de l'article}

Ce texte est une tentative pour opérer une transformation radicale de la problématique qui prétend appréhender l'altérité en dégageant et restituant la véritable matrice originaire qui la génère inéluctablement et la fonde ; matrice qui régit, selon moi, la constitution et le fonctionnement de notre corporéité même puisqu'elle innerve et détermine tout processus sensoriel et moteur. C'est ce que j'entends essayer de démontrer en soulignant, d'une part, les différents niveaux et les diverses modalités qu'elle revêt dans l'expérience humaine ; d'autre part et surtout sa manifestation primordiale et son orchestration esthétique illimitée dans l'acte de danser. 

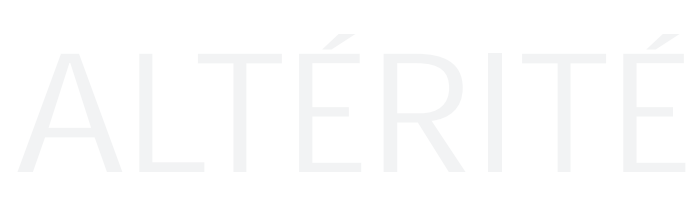

\title{
L'ALTÉRITÉ O RIG IN AIRE OU LES MIRAGES FO NDATEURS DE L'IDENTITÉ
}

\author{
MICHEL BERNARD
}

Tout d'abord un constat. Le mot «altérité» dérive du radical latin «alter» traduit par «autre», qui est donc à la fois adjectif et substantif puisque signifiant aussi bien le caractère d'étrangeté d'une perception que le sujet ou l'objet auquel on le rapporte. Ainsi le terme d' «altérité», qui a été forgé à partir de ce radical, est une catégorie abstraite qui ne désigne pas une chose existante ou un référent empirique déterminé, mais un rapport, une relation suscitée et engendrée par une exigence de connaissance et par là un désir d'identification en vue de se garantir contre l'imprévu, d'appeler une reconnaissance et d'acquérir légitimement une place ou un pouvoir, bref une sécurisation dans l'espace, le temps et la société. Autrement dit, ce concept est toujours fondamentalement une réponse et, qui plus est, une réponse négative, à une question, à savoir la quête d'identité, c'est-à-dire la volonté d'emprise et de maîtrise que constitue tout acte cognitif: celle de la réduction de tout événement insolite et déstabilisant au déjà vu et pensé, senti et conçu et a fortiori nommé, bref, selon la terminologie philosophique, au «Même». En ce sens, l'altérité apparaît toujours au premier abord comme un phénomène adventice, contingent et dangereux qui menace plus ou moins explicitement l'identité profonde et permanente, l'essence spécifique et individuelle qui est censée nous constituer et nous définir.

Toute la tradition philosophique occidentale l'atteste depuis les tentatives grecques de réduction du multiple à l'un, du mouvement à l'immuable, de l'apparence à l'être, en passant par la croyance chrétienne en la coexistence et mieux encore la communion de la diversité des créatures dans l'harmonie du monde conçu par le Créateur, l'idée cartésienne d'une "res cogitans" souveraine qui permettrait la compréhension de tous les sujets ou l'hypothèse mathématique et métaphysique de la monadologie leibnizienne qui assure à la fois la distinction des substances et leur compossibilité et correspondance universelle; ou bien aussi la subordination kantienne de l'altérité phénoménale et empirique au pouvoir législatif des formes a priori de la sensibilité, de l'entendement et de la raison, jusqu'à la neutralisation hégélienne des forces d'opposition dans et par le mécanisme du jeu dialectique, tel que l'illustre la célèbre interprétation de la confrontation du maître et de l'esclave. En fait, comme l'a fort justement relevé Vincent Descombes ${ }^{1}$, il faut attendre la remise en cause de cet héritage philosophique par l'interrogation subversive de ceux 
que Ricœur appelle «les trois maitres du soupçon", à savoir Marx, Nietzsche et Freud, pour voir s'inverser la perception et l'évaluation de l'altérité qui désormais acquiert une totale, irréductible et primordiale positivité.

Mais il faut maintenant, selon moi, aller plus loin encore et opérer une transformation radicale de la problématique qui l'appréhende en dégageant et restituant la véritable matrice originaire qui génère inéluctablement et, par conséquent, fonde ce rapport à l'altérité; matrice qui régit la constitution et le fonctionnement de notre corporéité même puisqu'elle innerve et détermine son processus sensoriel et moteur. C'est ce que je vais tenter de démontrer ici en soulignant, d'une part, les différents niveaux et les différentes modalités qu'elle revêt dans l'expérience humaine; d'autre part, et surtout, sa manifestation primordiale et son orchestration esthétique illimitée dans l'acte de danser. $\mathrm{Si}$, comme je viens de le mentionner, l'altérité désigne non une chose en soi, mais le rapport entre un phénomène perçu et ma manière de le percevoir comme distinct sinon étranger à ma propre corporéité et à l'ensemble de mon environnement, on peut dire qu'elle s'offre nécessairement comme la conséquence ou l'effet d'un processus de différenciation qui travaille non seulement la totalité du monde vivant et matériel, mais plus radicalement la temporalité qui les habite et les affecte. Or précisément ce processus conjugue des modalités très diverses selon les niveaux où il se situe.

J'en distingue, pour ma part, sept principaux. Tout d'abord, bien entendu, le niveau physique qui laisse apparaître plusieurs types d'altérité concrétisés soit par la séparation matérielle stricte et évidente des choses dans l'espace, soit par la distinction de leurs attributs permanents, à savoir taille, forme, couleur, propriétés extrinsèques ou intrinsèques de leurs composants, soit encore par la diversification de ces mêmes attributs au cours des changements temporels. Autrement dit, il y a une première modalité générique d'altérité qui est celle de l'individuation physicochimique de la matière.

Or cette individuation se prolonge en se complexifiant par une seconde qui se manifeste cette fois au niveau biologique, à savoir l'individuation des êtres vivants: l'essence même de la vie, en effet, est de se dupliquer à l'infini dans la prodigalité tragique de la multiplication cellulaire et du processus reproducteur. Comme le dit François Jacob, «la reproduction sexuelle est une machine à faire autre ${ }^{2}$; machine qui trouve son moteur radical dans le mécanisme embryologique de "la gastrulation", c'est-à-dire cette sorte d'invagination précoce par quoi se forment des feuillets cellulaires superposés et du même coup les différenciations organiques fondamentales. Ainsi très récemment a-t-on mis à jour et commencé à explorer le fonctionnement étrange des cellules souches embryonnaires à l'origine de la constitution soit de la diversification des organes, soit de la spécification de chacun d'entre eux, soit de celle de la totalité de l'organisme lui-même par rapport non seulement à ceux des autres espèces, mais aussi à ceux de la même espèce. C'est là, comme le souligne F. Jacob, «le second volet du paradoxe» du vivant:

[...] la diversité ne concerne pas seulement les différences entre espèces. Elle s'adresse aussi aux individus d'une même espèce...

Au cours des vingt ou trente dernières années, la biologie n'a cessé de mettre en évidence ce qui, chez les espèces à reproduction sexuée les plus diverses, la nôtre en particulier, caractérise chaque individu. Différences immunologiques révélées d'abord par les greffes de peau ou d'organes, puis par l'étude des gènes qui déterminent la structure des molécules garnissant la surface des cellules ainsi que ceux commandant les mécanismes de rejet. Différences génétiques multiples mises en évidence par les comparaisons d'ADN d'individus variés, ce qui a conduit à préciser les empreintes génétiques de chaque individu, plus révélatrices que les empreintes digitales et mieux à même de repérer les responsables de crimes ou de paternité. Immunologie et génétique, poursuit Jacob, ont ainsi amplement démontré que, à l'exception des vrais jumeaux, chacun de nous est différent des êtres humains qui ont vécu, qui vivent ou qui vivront sur Terre. ${ }^{3}$

Bref, il y a une altérité biologique qui semble, par la finesse et la sophistication de son organisation, enrichir et opacifier davantage l'altérité purement physique et primordiale du Cosmos.

Or curieusement ces deux formes d'altérité primitive ne cessent de se continuer et s'orchestrer dans les niveaux d'évolution ultérieurs. Ainsi, à un 
troisième niveau, voit-on se manifester la diversité irréductible des psychismes par la disparité des comportements individuels et des personnalités qu'elle exprime. C'est là la fameuse thématique exploitée par les psychologues et psychanalystes de la genèse du Moi dans et par son opposition à l'Autre; thématique galvaudée qui a malencontreusement, à mes yeux, eu tendance non seulement à interférer et verrouiller à son profit la problématique générale de l'altérité, en subordonnant et neutralisant la spécificité de tous les autres niveaux et de leurs modalités propres, mais à occulter et masquer son processus moteur fondamental.

Ainsi a-t-elle plus particulièrement annexé et prédéterminé l'approche de deux types complémentaires d'altérité: l'un, sociologique, imposé par la complexité de l'organisation sociale; l'autre, proprement anthropologique, découlant de la différenciation culturelle. Toute société, en effet, implique, par définition, la reconnaissance de la coexistence d'individus distincts, qui s'acceptent dans leur singularité respective, avec la volonté de s'associer au sein de groupes institutionnels variés, auxquels ils confient la mission et l'autorité d'assurer l'harmonisation relative de leurs multiples différences et davantage encore la résolution de leurs conflits d'intérêts. Il n'y a donc de vie sociale qui ne soit révélatrice des oppositions profondes ou superficielles qu'elle prétend précisément unifier en les dépassant ou les transcendant dans une identité collective supérieure: oppositions ou antagonismes soit entre catégories socioprofessionnelles, soit entre classes, soit entre organisations politiques ou idéologiques, soit entre clubs ou associations, soit entre établissements publics ou privés, etc. Autant d'oppositions qui s'enracinent et se manifestent dans une altérité connexe et complémentaire, celle précisément de la différenciation de l'appartenance culturelle. C'est, comme nous le savons, cette altérité anthropologique que le célèbre Séminaire de 1974-75 sur l'identité, dirigé au Collège de France par Claude Lévi-Strauss assisté de J.M. Benoît, a essayé de cerner, inventorier et expliquer dans une vaste confrontation interdisciplinaire ${ }^{4}$; ou bien encore que Tzvetan Todorov, dans son livre Nous et les Autres, souhaite résoudre par «un humanisme bien tempéré " 5 . En fait, cette altérité réside avant tout dans les multiples aspects ou dimensions revêtus par le rattachement ethnique envisagé dans sa spatialité et sa temporalité, à savoir les différences d'inscription topologique ou d'implantation territoriale et celles de son évolution historique depuis la spécificité de ses structures de parenté, d'organisation et de fonctionnement familial, ses modes d'accès et d'initiation au langage et de participation aux mythes jusqu'à la singularité des techniques de survie, des pratiques religieuses, ludiques, sexuelles, artistiques, des modes de gestion du pouvoir et de hiérarchie interne, etc. Bref, cette diversification culturelle est telle qu'elle semble remettre en cause la possibilité d'une définition d'une essence humaine une et identique, permanente et universelle.

Or certains néanmoins, comme Julia Kristeva, par exemple 6 , s'efforcent de la maintenir et garantir en cherchant à lui trouver un fondement dans les mécanismes psychologiques, et plus exactement psychanalytiques, qui gouvernent et animent l'ensemble de ce tissu relationnel du travail culturel. D'où une appréhension parfois quelque peu biaisée et tronquée de cette double altérité sociologique et anthropologique, réduite au statut d'épiphénomènes du processus comportemental d'adaptation à un environnement écologique, physique, vital et humain. Cette forme de psychologisation tend ainsi à gommer et méconnaître, selon moi, les deux derniers niveaux et modes d'altérité les plus profonds et plus significatifs: celui qu'aborde et problématise la philosophie et celui, conjoint, que dévoile et explore la perception esthétique.

Toute la philosophie se confond, en effet, avec un effort ininterrompu, laborieux et angoissant pour conceptualiser l'expérience déconcertante du changement et du mouvement qui s'offre comme le mariage étrange d'un être et d'un non-être, d'une altérité dans une identité, d'une différence dans une répétition. D'où, par exemple, les deux explications célèbres avancées successivement par Platon et Aristote: d'une part, la dialectique entre les deux mondes sensible et intelligible, les apparences et les Idées, telle que l'illustre l'allégorie de la caverne; 
d'autre part, l'hypothèse hylémorphique, c'est-à-dire l'assimilation de toute réalité à une union d'une matière et d'une forme, d'une essence et de ses accidents et au passage de la puissance à l'acte qui, selon Aristote, permet de rendre compte de la différenciation du particulier dans l'universel. Deux explications qui n'ont cessé de hanter toute la tradition philosophique ultérieure et de résonner sur elle. Il est significatif à ce propos de noter que toute la philosophie française contemporaine, et plus particulièrement dans ses quarante-cinq années les plus productives, de 1933 à 1978, a pu être appréhendée et exposée par Vincent Descombes sous cet éclairage thématique fondamental: «le même et l'autre", qui est le titre d'un livre ${ }^{7}$ où sont regroupés les études hégéliennes de Kojève et Sartre, la phénoménologie merleau-pontyenne, le structuralisme des années soixante, les critiques althussérienne et foucaultiste de l'Histoire, la philosophie derridienne de la différance, la schizoanalyse de Deleuze et Guattari, l'économie libidinale de Lyotard et le nietzschéisme de Klossowski. J'ajouterais aussi, à titre de confirmation, s'il en fallait une, d'une part, que toute l'œuvre de Levinas s'ordonne autour du concept d'altérité dont l'identité ne serait, dit-il, que "la maladie», comme l'attestent ces deux derniers ouvrages: Autrement qu'être ou au-delà de l'essence (1988) et Le Temps et l'Autre $(1993)^{8}$; d'autre part, que J. Baudrillard a cru pouvoir, dans son travail d'Habilitation, synthétiser la totalité et la diversité de sa recherche sous la dénomination, ô combien révélatrice: L'Autre par lui-même ${ }^{9}$, ce que vient corroborer l'argumentation de son dernier livre L'Échange impossible 10 qui est, au fond, la description et l'analyse de ce qu'il appelle "le jeu de l'altérité» dont je parlerai plus loin. Bref, toute la démarche philosophique se déploie et se focalise autour de l'altérité dans la mesure même où elle constitue et habite le processus temporel qui est, comme l'a fort justement souligné M. Merleau-Ponty ${ }^{11}$, le noyau ou le cœur de toute réflexion.

Aussi n'est-il pas étonnant que le septième et ultime niveau et mode d'altérité se situe dans le champ esthétique, c'est-à-dire celui de l'exploration et de l'exploitation du sentir par l'Art qui n'est, en réalité, que la manière de jouer à l'infini avec cette temporalité radicale qui parcourt et anime le réseau chiasmatique de notre corporéité. Toute création artistique n'est-elle pas, en effet, à la fois la reconnaissance de l'altérité primordiale du matériau sensible qu'on appréhende et veut travailler, l'éclosion de celle, conjointe, du projet fictif de cette transformation et, a fortiori, l'avènement décisif de celle de la production effective de l'œuvre? Autrement dit, quelle que soit la nature de la sensation organique (visuelle, auditive, tactile, olfactive, gustative ou kinesthésique) qui le déclenche et le véhicule, le travail de l'artiste est toujours l'expérience de l'altérité immanente à la temporalité de son désir et de son acte créateur.

Bien plus, la corporéité qu'il sollicite et utilise ne suscite-t-elle pas, à son tour, indépendamment de la différenciation biologique anatomique et physiologique qui la constitue, et par le seul déploiement spectaculaire de sa mise en jeu kinesthésique, deux autres types d'altérité que manifestent précisément le théâtre et davantage encore la danse? Il y a, en effet, dans l'acte de danser, un premier type d'altérité que j'appellerai "performatif", puisqu'il est inhérent au seul mouvement en tant que tel. Comme l'a fort bien noté Trisha Brown, le mouvement du danseur ou de la danseuse implique une sorte de "distribution démocratique» de la corporéité, c'est-à-dire une manière de jouer et d'orchestrer ses différentes parties par un processus de recouvrement clandestin ou d'articulation secrète de mouvements simultanés virtuels et subtils: «j'altère, dit-elle, le sens d'un mouvement $a$ en le pénétrant d'un mouvement $b{ }^{12}$. Bref, tout processus dynamique présuppose une altérité immanente au dispositif synergique de l'effectuation motrice.

D'où, conjointement, un second type d'altérité que je qualifierai cette fois de "théâtral", dans la mesure où le ou la chorégraphe peut, dans sa composition, jouer ou faire jouer plus ou moins habilement les différents éléments de ce dispositif dans une finalité spectaculaire de théâtralisation du mouvement dansé. C'est, semblet-il, comme l'a fort bien vu André Lepecki, ce que fait Meg Stuart en 1995 dans son solo Xxx. For Arlene and Colleagues à la Judson Church de New York: 
[...] elle traite, dit-il, son corps comme un collectif et assigne à chaque membre, chaque moitié du corps, chaque partie de son visage, les traits d'un personnage individuel. En comprimant la variété ethnographique dans sa seule présence, elle produit un effet tout en puissance et en transparence. ${ }^{13}$

Ainsi on constate que la danse dévoile, explore et exploite, par-delà la diversité statique des structures organiques, des modes d'altérité corporelle qui paraissent constituer, en quelque sorte, les racines profondes de tous les niveaux que j'ai tenté précédemment d'inventorier et évoquer. Il n'en demeure pas moins que de nombreux auteurs ont quelque peu négligé cet enracinement esthétique et plus spécifiquement chorégraphique et se sont ingéniés à interpréter leur diversité selon des perspectives et modèles réductionnistes en fonction d'une méthodologie dominante et/ou du choix d'un champ épistémologique privilégié, ce qui, à mes yeux, a malheureusement contribué à occulter et même dénaturer l'approche et a fortiori l'éclaircissement de ce problème fondamental et difficile de l'altérité. C'est ce que je voudrais maintenant, dans une seconde partie, essayer de démontrer en proposant in fine, au terme de cette analyse, une hypothèse personnelle susceptible de le résoudre et surtout de mieux faire comprendre, d'approfondir et enrichir le travail du danseur ou de la danseuse.

La première forme d'interprétation qui a prévalu et a cru pouvoir surmonter les difficultés et les insuffisances de l'altérité est bien entendu celle qui a été conçue, théorisée et transmise par les fondateurs de la pensée occidentale et qu'on regroupe habituellement sous l'étiquette de «tradition classique». Il s'agit, comme on le devine, du modèle ontologique, tel qu'on le rencontre dans la philosophie grecque de Platon et d'Aristote et chez leurs héritiers plus ou moins fidèles, à savoir principalement les scolastiques du Moyen Âge et, dans une optique différente, Descartes et ses trois principaux disciples: Spinoza, Malebranche et Leibniz. Ce modèle consiste, ainsi que nous l'avons signalé succinctement dans notre problématique, non seulement à amalgamer et réduire toutes les formes d'altérité à un seul et unique statut de nature purement métaphysique, celui d'apparence contingente, mais aussi et surtout à assigner à ces apparences le destin inéluctable, la nécessité de se fondre dans l'identité de l'être. Cette identité ontologique constitue donc la norme absolue et la finalité de toute différenciation et a fortiori de toute hétérogénéité: est autre ce qui trouble, pollue, perturbe, fragilise et appauvrit l'être par sa négativité, son instabilité, sa mutabilité. L'autre n'est pas, mais devient et paraît. À la limite, l'Un peut être dit, comme le fait Plotin, au-delà de l'Être lui-même, par le devenir potentiel qui le mine. Bref, l'altérité, dans cette perspective, dissout toutes ses modalités de différenciation ou variation immanente et se dilue elle-même dans les mirages du devenir de l'Être comme puissance souveraine.

Mais précisément ces mirages en sont-ils vraiment ou plus exactement ne faut-il pas rappeler et souligner qu'ils apparaissent comme tels pour une conscience qui ne peut pas ne pas les appréhender comme des êtres tout aussi présents et réels qu'elle-même, en un mot, comme des phénomènes et non comme des apparences inconsistantes et fugitives? C'est en tout cas la question que se pose Husserl qui note, dans ses Méditations cartésiennes ${ }^{14}$, que l'expérience du monde et des autres n'est pas celle de «reflets» éphémères, mais d'une opération de constitution simultanée d'une conscience percevante et d'un phénomène perçu, indéfectiblement liés dans un même tissu ontologique qu'il appelle «intentionnalité». En d'autres termes, l'expérience originaire de l'être est intersubjective et non monadique ou solipsiste. Telle est la seconde interprétation dite phénoménologique du problème de l'altérité qui, sans rompre ou renier la perspective ontologique, la décentre et la module radicalement. Elle a été, comme chacun sait, reprise et développée par M. Merleau-Ponty dans sa Phénoménomogie de la perception où, insistant sur cette coexistence primordiale et, comme telle, transcendantale du Moi et des Autres au sein de l'intentionnalité, il ne craint pas d'affirmer que «l'existence d'autrui ne fait difficulté et scandale» que "pour la pensée objective» mise en œuvre par les adultes et en revanche complètement absente chez les enfants ${ }^{15}$. Dans le même sillage phénoménologique, 
E. Levinas va encore beaucoup plus loin dans la mesure où il récuse non seulement l'hégémonie ou le monopole de la référenciation ontologique, mais aussi la reconnaissance de l'intentionnalité comme vecteur déterminant. Pour Levinas, c'est l'accueil passif de l'Autre qui constitue ma subjectivité, la fait naittre, l'habite, la temporalise et, par conséquent, rend caduque toute préséance ontologique d'une prétendue identité d'un Moi comme conscience intentionnelle. Il n'y a donc pas, à ses yeux, de vis-à-vis dans la violence implicite et feutrée d'une frontalité, mais l'expérience humaine de l'ouverture à "un Visage», c'est-à-dire à une expression et une parole et non à la réalité objective pesante et contraignante d'un faciès physique, d'une physionomie organique. L'altérité ne surgit alors que comme appel à un échange et un dialogue dans le champ de l'être, bref à une relation éthique. Elle ne se situe plus dans le champ de l'être, mais, comme l'énonce le titre d'un des derniers livres de ce philosophe, dans l'Autrement qu'être ou au-delà de l'essence ${ }^{16}$. Beaucoup plus que chez Husserl, cette approche de l'altérité semble ainsi se démarquer fondamentalement du modèle ontologique proposé par les Grecs en déplaçant à la fois son centre de perspective, son cadre de référence et son sens. Néanmoins, en dépit de cette réorientation, le modèle phénoménologique levinassien reste fidèle à la prescription husserlienne: il «exclut la métaphysique naïve, mais non la métaphysique en général ${ }^{17}$ à laquelle il croit nécessaire seulement de conférer une assise éthique. La problématique de l'Autre chez Levinas tout comme chez Husserl continue de s'inscrire dans le champ relationnel d'un Sujet, si défaillant, exposé et vulnérable soit-il, en quête de sens, ce qui implique la reconnaissance tacite de la légitimité d'une forme de questionnement centrée sur l'exigence d'élucidation du phénomène de la conscience de l'Autre et, par là, qu'on le veuille ou non, comme l'a souligné Derrida, d'un a priori subjectif en tant que point de vue transcendantal ${ }^{18}$. En somme, quels que soient la forme qu'on lui donne et l'usage qu'elle suscite, l'interprétation phénoménologique du statut de l'altérité reste tributaire du postulat du primat d'une conscience interrogative et de sa visée humaniste, ce qui réduit inévitablement son champ d'intelligibilité et sa validité.

C'est ce que semblent avoir compris beaucoup de penseurs, en particulier dans les années soixante, c'està-dire à un moment crucial de l'essor de la linguistique avec l'école postsaussurienne (R.Jakobson, L. Hjelmslev, E. Benveniste) et des sciences humaines en général en tant que tentative d'explication précise et exhaustive de l'ensemble de notre environnement social. Face à un tel bouleversement épistémologique, ils jugent nécessaire, en effet, de rompre avec l'optique purement métaphysico-éthique de la phénoménologie et son choix délibéré et exclusif d'une description, si rigoureuse soit-elle, du vécu empirique de la relation intersubjective. Mieux encore, ils se proposent d'en inverser le centre de perspective en le situant désormais non plus dans le Sujet ou l'Ego, mais dans l'objet qui, bien loin d'être un simple vis-à-vis phénoménal, est envisagé comme réalité organisée ou plus exactement ensemble relationnel isomorphe, bref comme structure. D'où un nouveau modèle au sens strict parce que formel de l'altérité, le modèle structuraliste. Ainsi, à la naïveté du regard subjectif revendiquée par la conscience du phénoménologue, la conception structuraliste oppose la volonté scientifique de comparaison et de décryptage analytique et systématique des autres comme configurations signifiantes, c'est-à-dire totalités codifiées de signes, architectures sémiotiques analogues au langage théorisé par Saussure et ses disciples. Dans cette optique, l'Autre n'est donc plus appréhendé par et pour son sens, comme médium d'une expérience, d'une expression et d'une communication, mais en tant que composition réglée, syntaxe et rhétorique d'unités signifiantes qui seraient, pense-t-on, des invariants universels. Telle est, par exemple, la démarche de Lévi-Strauss et de son équipe, pour lesquels la différenciation des individus et des groupes qui composent toute société se réduit à celle des règles qui déterminent toutes leurs modalités d'échanges, aussi bien des femmes que des richesses et des messages proprement dits, en somme leur système de parenté, de gestion économique et de communication linguistique. S'il y a une altérité 
sociale et anthropologique, c'est celle, par conséquent, des structures comme grammaires ou codifications distinctes qui régissent l'apparente diversité collective et ethnique; structures qui ne dépendent pas de l'émetteur comme Sujet intentionnel, qui précèdent le message lui-même et son désir de sens et, en définitive, se manifestent dans la seule extériorité et priorité du signifiant par rapport au signifié, ce qui fait dire à Lacan que, comme le rappelle V. Descombes ${ }^{19}$, «le discours fonde le lien social», devenant par là même un pur lien symbolique. Ainsi s'explique la place prépondérante accordée par Lévi-Strauss aux mythes: bien loin d'être l'expression du vécu de la conscience collective du récitant qui seule lui donnerait son sens, le mythe est, en effet, avant tout, aux yeux de ce philosophe, un récit avec une forme narrative spécifique qui préexiste à la narration et dont on peut déterminer le code. En le comparant dès lors à ceux d'autres récits analogues, on est en mesure, selon lui, d'en dégager une série d'invariants présents dans toutes les sociétés, si différentes soient-elles et indépendamment de leur situation géographique et de leur histoire. L'apparente irréductibilité des ethnies étudiées est donc sous-tendue par l'identité relative des structures, ce qui revient à reconnaître une relativisation du discontinu ou, si l'on préfère, une similitude et continuité des codes par-delà l'écart différentiel comme donnée insurmontable.

Dans cette hypothèse, écrit Lévi-Strauss, l'identité est une sorte de foyer virtuel auquel il nous est indispensable de nous référer pour expliquer un certain nombre de choses, mais sans qu'il ait jamais d'existence réelle.

Ainsi croit-il pouvoir proposer une solution à l'antinomie qui lui est classiquement objectée: [...] vous voulez étudier des sociétés complètement différentes, hi dit-on, mais, pour les étudier, vous les réduisez à l'identité [...] cette solution n'existe, répondill, que dans l'effort des sciences humaines pour dépasser cette notion d'identité, et voir que son existence est purement théorique: celle d'une limite à quoi ne correspond en réalité aucune expérience. 20

Il apparaît donc que l'approche structuraliste se veut essentiellement scientifique et par là procède d'une quête de la raison, bien plus d'une raison élargie dans la mesure où elle vise à "comprendre, comme l'écrit Merleau-Ponty ${ }^{21}$, ce qui en nous et dans les autres précède et excède la raison» et que Lévi-Strauss lui-même appelle "une sorte de superrationalisme»22. Autrement dit, il s'agit d' «apprivoiser l'élément brutal de l'existence, d'assimiler l'hétérogène, donner sens à l'insensé, de rationaliser l'incongru, bref, traduire l'autre dans la langue du même» ${ }^{23}$, même si celui-ci est purement asymptotique. Une telle tâche implique, par conséquent, comme on le voit, un double postulat que certains peut-être jugeront quelque peu contradictoire: d'une part, celui de la légitimité d'une visée universalisante et, du même coup, de la croyance en l'existence d'invariants universels; d'autre part, celui du monopole hégémonique du code linguistique comme unique référence et seul fondement épistémologique et axiologique, ce qui destitue et invalide du même coup l'Homme comme producteur privilégié de sens et critère exclusif de valeur, en un mot l'Humanisme. Ainsi l'étrange collusion de cet universalisme transcendantal et de cet antihumanisme réduit-elle l'Histoire, jusqu'ici prônée comme l'œuvre spécifique de l'Homme, à un mythe, le mythe occidental par excellence, celui que va précisément déconstruire Michel Foucault en dévoilant la structure épistémologique ou, selon sa terminologie, «l'épistémè» qui la rend possible et la fait naître.

Or ce double postulat autant que la critique de l'Histoire qui en résulte sont loin d'être évidents et en tout cas font problème. Bien plus, la démarche structuraliste qu'ils autorisent n'offre-t-elle pas un certain paradoxe relevé et dénoncé à juste titre par Vincent Descombes? En effet, d'une part, elle proclame, à l'encontre de la Phénoménologie, que le signifiant n'est pas au service du Sujet ou, si l'on préfère, de la soumission de l'Homme aux systèmes signifiants constitués par l'universalité intemporelle de la structure linguistique; d'autre part, et d'une façon quelque peu surprenante, elle démontre la légitimité de cette assertion

[...] en puisant ses concepts dans la théorie de l'information, c'est-à-dire dans une pensée d'ingénieurs dont le voeu est, comme l'indique le mot "cybernétique» dont ils ont fait leur titre 
scientifique, de donner à l'être humain le contrôle de toute chose grâce à une meilleure maîtrise de la communication. 24

Pour résoudre ou surmonter un tel paradoxe, ne convient-il pas dès lors de rectifier l'usage et l'interprétation de cette théorie de l'information en conciliant l'organisation de la dimension structurale et l'intégration du statut d'un sujet producteur et responsable, en d'autres termes, l'identité épistémologique et l'altérité ontologique? C'est ce que tentent d'effectuer certains chercheurs en proposant un quatrième modèle d'intelligibilité de cette identité et de cette altérité qui a précisément pour caractéristique non de les dissocier en une relative exclusion comme dans le modèle structuraliste, mais au contraire de les joindre ou enchaîner indéfectiblement toutes deux comme phases du processus ontologique lui-même en tant que devenir ou dynamique de production de l'individu, en un mot, du processus d'individuation. Ce modèle, que j'appellerai systémique, est celui qui est invoqué, expliqué et justifié avant tout par Gilbert Simondon dans un remarquable ouvrage intitulé L'Individu et sa genèse physico-biologique ${ }^{25}$.

Contrairement aux trois approches précédentes, qui toutes présupposent et partent d'une altérité constituée et donnée dans la réalité de l'individu comme entité distincte soit physique, soit biologique, soit psychologique et sociale ou de tout autre ordre, G.Simondon postule fondamentalement que le principe d'individuation est antérieur à l'individu luimême, autrement dit que celui-ci n'a aucun privilège ontologique, mais bien au contraire qu'il reçoit son être du devenir qui le génère. Il faut donc inverser la démarche admise jusqu'ici et connaître l'individu à travers l'individuation, c'est-à-dire le processus de différenciation comme formation des altérités multiples, ce qui revient, en fait, à reconnaître que la genèse est constitutive de l'être lui-même, sa dimension primordiale et non son opposé comme le soutient la Philosophie traditionnelle. En d'autres termes, si Simondon recourt à «un postulat de nature ontologique» ${ }^{26}$, celui-ci change de modalité et par là de signification: l'être se fait devenir et plus précisément "ontogenèse de l'individu» comme structure à la fois identique et autre qui n'en est donc qu' «une phase " 27 . Ainsi, avec cette hypothèse, Simondon à la fois réinterprète à sa manière et relativise encore davantage ce concept de structure qui constitue la pierre angulaire de l'épistémologie lévistraussienne. Il la réinterprète et la relativise radicalement, en effet, d'une part, en l'inscrivant dans le cadre du fonctionnement d' «un système» organisé beaucoup plus vaste et plus mobile ou, selon sa propre expression, «métastable»; d'autre part et conjointement, en expliquant cette métastabilité même par la nature essentiellement énergétique de ce système. Autrement dit, la structure à la fois perd son privilège de fondement théorique exclusif et change de référent épistémologique: sa dimension systémique n'est plus calquée sur celle de la permanence du code linguistique ou sémiologique, mais sur celle de la dynamique du processus énergétique qui régit la totalité de l'univers. Elle n'est, en fin de compte, qu'un état précaire d'équilibre ou «un cas de résolution apparent et passager du système métastable» de l'évolution de l'énergie cosmique.

Cette métastabilité systémique se définit, en effet, selon Simondon, par «trois notions fondamentales: celle d'énergie potentielle, celle d'ordre et celle d'augmentation de l'entropie» ${ }^{28}$. Contrairement aux Anciens qui opposaient mouvement et repos, stabilité et instabilité, il faut, dit-il, envisager l'être comme une énergie primitive et originelle soumise à des tensions constantes et qui ne cesse de se dédoubler dans un couple ou une polarité individu/milieu. D'où de multiples phases d'équilibre qui constituent autant d'ordres spécifiques où l'énergie se transforme en se dégradant inexorablement. Ainsi, par cette métastabilité énergétique immanente, le processus d'individuation tend vers trois ordres ou niveaux successifs de complexification: le niveau physique, tout d'abord, où on voit, grâce aux théories complémentaires des champs et des corpuscules tout comme celles des quanta et de la mécanique ondulatoire qui illustrent en quelque sorte «un régime de préindividuel», surgir des états de système macroscopique comme les cristaux; le niveau biologique ensuite qui perpétue dans le vivant ce 
premier état d'individuation par une «résonance interne" indéfinie, une sorte de "théâtre d'individuation", selon l'heureuse expression de Simondon 29.

Il y a dans le vivant, écrit-il, une individuation par l'individu et non pas seulement un fonctionnement résultant d'une individuation une fois accomplie, comparable à une fabrication.

Bref, le vivant se modifie lui-même en modifiant sa relation au milieu: il est "système individuant et système $s^{\prime}$ individuant» ${ }^{30}$. Un troisième niveau, à la fois et indissolublement psychique et collectif, se manifeste enfin dans

[...] la poursuite de l'individuation vitale chez un être qui, pour résoudre sa propre problématique, est obligé d'intervenir lui-même comme élément du problème par son action, comme sujet; le sujet peut être conçu comme l'unité de l'être en tant que vivant individué et en tant qu'être qui se représente son action à travers le monde comme élément et dimension du monde. 31

Or précisément cet être psychique ne peut résoudre sa propre problématique, comme nous le disions, qu'en l'insérant dans l'individuation collective qui constitue, d'une certaine façon, l'extériorité de celle conquise intérieurement par le sujet. Ces deux individuations réciproques définissent ainsi une catégorie de «transindividuel»32, où le vivant s'éprouve dans la mouvance et la précarité des relations adaptatives de l'individu au milieu: d'une part, par le couple primitif de la sensation et du tropisme; d'autre part, par le couple cognitif de la perception et de la science; et enfin simultanément par celui de l'affectivité et de l'émotivité en tant que résonance interne de ce processus. En somme, pour Simondon, il n'y a plus de réalité unique et homogène, mais un système

foncièrement métastable comme opération perpétuelle d'individuation, c'est-à-dire de production d'ordres disparates en tension permanente, ce qui précisément, à ses yeux, définit la notion d' «information»: «elle est, écrit-il, la signification qui surgira lorsqu'une opération d'individuation découvrira la dimension selon laquelle deux réels disparates peuvent devenir système " 33 . Ainsi, contrairement à l'acception technologique ou cybernétique commune qui la réfère aux signaux, elle ne désigne pas une chose donnée, un message émis réellement, mais seulement "une exigence d'individuation", "un changement de phase d'un système». Du même coup, cette notion d'information se substitue à celle de Forme ou Gestalt en tant que structure constituée qui ne correspond, elle, qu'à un état du système ${ }^{34}$.

Comme on le voit, le modèle systémique proposé par Simondon implique donc une conception de l'être non plus substantielle, mais purement relationnelle: il n'a plus d'identité, mais ne cesse, écritil, de «se déphaser par rapport à lui-même, de se déborder lui-même de part et d'autre de son centre " 35 . C'est ce qu'il appelle "la transduction", c'est-à-dire, concrètement,

[...] une opération physique, biologique, mentale, sociale par laquelle une activité se propage de proche en proche à l'intérieur d'un domaine, en fondant cette propagation sur une structuration du domaine opérée de place en place: chaque région de structure constituée sert à la région suivante de principe de constitution, si bien qu'une modification s'étend ainsi progressivement en même temps que cette opération structurante.

En définitive, la transduction s'offre comme «une structure réticulaire amplifiante", «une individuation en progrès » ${ }^{36}$ qui, tout comme la dialectique hégélienne, suit l'être dans sa genèse. Mais, en revanche, à l'inverse de celle-là, la transduction ne fait pas apparaître le négatif comme seconde étape: elle le suppose, au contraire, «immanent dans la condition première sous forme ambivalente de tension et d'incompatibilité», en tant que potentiels appelés à s'individuer, donc à se convertir en structures positives. Par là même aussi,

[...] à la différence de la démarche dialectique, la transduction ne suppose pas l'existence d'un temps préalable comme cadre dans lequel la genèse se déroule, le temps lui-même étant solution, dimension de la systématique découverte: le temps sort du préindividuel comme les autres dimensions selon lesquelles l'individuation s'effectue. ${ }^{37}$

Néanmoins, à y bien regarder, il s'agit là, me semble-t-il, d'une conception tout aussi totalisante que 
celle de Hegel: la problématique de l'altérité s'inscrit dans une vision systémique qui est intégrative, par essence, et, par conséquent, dissout nécessairement l'opacité et l'hétérogénéité aléatoire de toute expérience quelle qu'elle soit, réduite à un statut de phase d'un devenir préréglé, d'une genèse théoriquement ou épistémologiquement programmée. En ce sens, tout en surmontant les contradictions internes de l'approche structuraliste, ce modèle interprétatif ne peut répondre aux questions soulevées par une telle expérience envisagée en elle-même et nous invite à la sonder davantage. Mais il est tout aussi indéniable, en revanche, qu'elle nous ouvre plusieurs pistes intéressantes, quatre principalement qui seront choisies et exploitées par d'autres modèles: la première est bien évidemment celle de considérer le devenir comme dimension fondamentale de l'être, autrement dit, d'en faire une ontogenèse. La deuxième est de caractériser précisément cette ontogenèse par un mécanisme de dédoublement ou de déphasage ininterrompu, par lequel chaque structure se prolonge dans un réseau indéfini de structures nouvelles de plus en plus différenciées, bref dans un processus d'individuation continuée. D'où aussi une troisième piste connexe qui consiste à envisager ce mécanisme d'individuation comme "une tension entre deux réels disparates", qui est l'information elle-même désormais étrangère à toute finalité technologique de transmission, en un mot, à définir le devenir ontologique comme tension permanente. Enfin, quatrième piste encore plus prometteuse parce que plus radicale, cette tension implique l'existence d'un fonctionnement énergétique généralisé en changement perpétuel: l'ontogenèse du même et de l'autre est un système énergétique métastable, en tant que structure réticulaire amplifiante. Or, comme je l'annonçai précédemment, ces quatre pistes ont connu divers prolongements ou développements parfois inattendus et surprenants, en tout cas indépendants de la vision systémique et épistémologique de Simondon. Ils se manifestent plus particulièrement dans trois autres modèles qui viennent utilement compléter et enrichir les quatre premiers qui ont été évoqués.
Ainsi je crois pouvoir distinguer un cinquième modèle interprétatif du phénomène d'altérité dans deux approches apparentées en tant qu'issues toutes deux du regard psychanalytique, à savoir la théorie d'ethnopsychanalyse complémentariste de Georges Devereux et la conception néofreudienne de l'entredeux avancée par Daniel Sibony ${ }^{38}$. Bien que différentes dans le discours et l'argumentation, ces deux approches se caractérisent, en effet, par un même souci de souligner la dimension inéluctable, irréductible et forte du lien ou de la relation qu'implique toute reconnaissance d'altérité: elles constituent donc deux prospections singulières et deux approfondissements parallèles de la deuxième et surtout troisième pistes simondonniennes mentionnées plus haut, à savoir, à l'intérieur du mécanisme de dédoublement, la mise à jour de la tension entre "deux réels disparates" immanente à tout processus d'individuation. En ce sens, je serais tenté de qualifier ce modèle de tensionnel ou interpolaire. Or cette tension revêt, chez Devereux, une forme particulière: elle découle de l'importance centrale qu'il accorde à la notion de «double». Le corps, à ses yeux, ne peut se comprendre qu'accompagné de ses doubles qui permettent de cerner ses frontières et de déterminer son identité en rapport avec une altérité. Il y a ainsi une grande diversité de doubles corporels: double métonymique (la partie pour le tout, le tout pour la partie); double métaphorique en tant qu'analogique; double spéculaire (portrait, ombre, écho, odeur, parfum); double symétrique (qui appartient, par exemple, à l'autre sexe ou à un autre monde); double identique (clone, sosie, jumeau); double par inversion ou réversibilité comme association du contenant et du contenu, du noyau et de l'écorce, du dedans et du dehors, du plein et du creux, du haut et du bas, de la gauche et de la droite, de l'avant et de l'arrière, du rétrécissement et de l'expansion; également double psychique qui résulte de l'introjection de la culture; et conjointement double culturel par projection de l'inconscient, et bien d'autres encore. Autant de doubles qui sont étudiés et servent d'outils privilégiés à l'ethnopsychiatrie de Devereux et de ses disciples comme Tobie Nathan ${ }^{39}$. En fait, dans cette optique, 
prévaut l'idée que toute forme d'altérité ou de différenciation, si minime soit-elle, obéit à l'exigence et même l'impératif de complémentarité, c'est-à-dire non de l'exclusion ou, à l'inverse, de la réduction, mais d'une force attractive de débordement et d'enrichissement, de fécondation réciproque: le complémentarisme ethnopsychanalytique apparaît, par conséquent, plus généralement comme un choix méthodologique qui régit non seulement les sujets ou a fortiori les objets, mais l'ensemble du champ théorique des disciplines qui les étudient.

Or il est remarquable, à ce propos, qu'une telle visée généralisante et à caractère méthodologique se retrouve dans l'approche également d'inspiration psychanalytique de Daniel Sibony. Néanmoins, chez celui-ci, l'accent est déplacé de la complémentarité du double vers l'espace et la dynamique de «la coupurelien» de l'entre-deux. En effet, selon lui, le concept de différence avec, dit-il, «ses sous-produits appréciables, la différance avec a inventée par Derrida et le différend employé par J.-F. Lyotard", ne suffit plus pour comprendre les divers phénomènes d'altérité de sexe, de race, d'âge, de nationalité, de langue, de culture, de profession et même d'identités multiples. Il faut aussi prendre en compte «l'espace où les deux termes semblent convoquer l'origine pour s'expliquer avec elle et pour que puisse s'élaborer un passage entre deux» 40 . Aux yeux de Sibony, «l'origine, ce n'est pas seulement là d'où on vient», mais ce sur quoi on bute et ce qui anime nos déplacements, une limite indépassable qui «induit des voies de passage». Ainsi, par exemple, écrit-il,

[...] il n'y a pas de langue-origine ni d'origine des langues, mais

les effets de l'origine viennent se jouer entre deux langues; il n'y a

pas La Femme, mais le fantasme qu'elle représente vient se jouer entre deux femmes; il n'y a pas La place mais sa question se joue dans le déplacement, l'entre-deux places; il n'y a pas la Vie ou la Mort mais ça se joue entre vie et mort; il n'y a pas d'Identité mais ça se joue dans l'entre-deux-identités et les mutations de l'origine. L'origine, ajoute-t-il, est un retrait qui conditionne l'entre-deux-traits. Elle se retire des entre-deux qu'elle implique et déclenche, et que son retrait conditionne. C'est pour cela que les entre-deux sont des figures de l'origine - des dissipations de l'origine. 41
En d'autres termes, l'entre-deux est une sorte d'«opérateur» dynamique qui travaille l'expérience étrange de la rencontre entre deux entités qui, impulsées par le fantasme d'une unité originelle, ne cessent de passer l'une par l'autre, de se confondre, de se détacher, de revenir et en même temps de s'éloigner.

L'origine, comme l'horizon, dit Sibony, nous suit quand on la

fuit, s'éloigne quand on y vient, et ses éclipses ou retours se marquent non par une donnée pure, unique, mais par deux moments, deux instances, entre lesquelles on est pris, on se retrouve pris, souvent à son insu.

Bien entendu, la fin de cette citation laisse deviner que toute cette argumentation et le modèle qu'elle tend à promouvoir procède ou découle de l'hypothèse psychanalytique de l'inconscient comme régisseur caché et en même temps ambivalent de toute notre vie. Sibony en tout cas reconnaît indirectement le poids de cette hypothèse sur sa propre approche de l'altérité lorsqu'il écrit:

[...] il se pourrait que la grande trouvaille de Freud tienne dans

cette mauvaise nouvelle qu'il a apportée aux humains: l'homme est porté par quelque chose de lui qui lui est étranger; il n'est pas intégré à lui-même; et ce à cause d'un écart intrinsèque qui s'appelle «inconscient». Cela n'empêche pas, continue Sibony, d'essayer d'intégrer, de s'intégrer, à condition d'être en mesure de supporter que ça échappe. 42

Et on sait que Julia Kristeva, de son côté, a largement exploité et orchestré ce thème dans son livre déjà mentionné au titre révélateur: Étrangers à nous-mêmes. Mais il est remarquable aussi et pour le moins assez surprenant que Sibony, lui, ait voulu conférer à cette origine, en soi purement métapsychologique, un statut plus général et en quelque sorte l'extrapoler à d'autres champs disciplinaires. Ainsi prétend-il l'appliquer légitimement aussi bien à la physique, avec l'exemple caractéristique de la vitesse de la lumière comme phénomène originaire dans la texture et la constitution de l'espace, que dans l'astronomie avec la théorie du big bang comme origine du temps et, par là, de l'entre-deux des successions, et dans les mathématiques enfin avec la théorie des ensembles dont l'origine se situe dans le tout de l'univers qui, 
par son retrait, permet aux autres de se compter comme ensembles et de se comparer ${ }^{43}$.

En fait, cette métaphorisation systématique de l'origine, dont l'identité n'est qu'une figure ou une prise de vue passagère par l'épreuve de l'entre-deux ${ }^{44}$, m'incite à penser qu'elle procède d'une mythologie souterraine, en l'occurrence ici chez Sibony, d'une herméneutique théologique de l'Ancien Testament et plus exactement de la Genèse, comme le laissent apparaître les cinq dernières pages de la conclusion de son livre ${ }^{45}$ : si l'entre-deux, comme il ne cesse de le répéter, est issu du partage de l'origine avec elle-même et avec l'Autre et, par conséquent, d'une coupure qui est en même temps lien, il se manifeste avant tout dans une certaine idée biblique de l'alliance.

En effet, écrit Sibony,

[...] il se trouve que c'est l'Ancien Testament qui le premier a mis en lumière cette idée: pour faire alliance on coupe en deux, on reconnaît la coupure voire la béance, ça ouvre l'espace d'un entre-deux à travers quoi passent le lien, ses transmissions, ses traductions et métamorphoses... et on renoue avec tout ça. L'entre-deux devient un espace de lien «entre l'un et entre l'autre». 46

Autrement dit, il paraît évident, à mes yeux, que le modèle d'interprétation du couple antinomique identité/altérité proposé par Sibony se fonde, par-delà la référence à la théorie freudienne de l'inconscient, sur une exégèse religieuse du texte sacré du Livre des Hébreux. Il neutralise ainsi l'interrogation soulevée par cette antinomie au profit d'une croyance feutrée ou masquée qui, d'une certaine façon, mutatis mutandis, fait écho à l'idéologie laïque, socialisante et humaniste du complémentarisme ethnopsychiatrique de Devereux. Bref, l'approche que j’ai appelée «interpolaire ou tensionnelle», focalisée entièrement sur l'espace et la dynamique de la relation avec l'autre, demande à être recentrée et éclairée dans ses véritables soubassements énergétiques, et plus exactement dans la seule matérialité de son apparition.

C'est précisément ce que tentent de faire Gilles Deleuze et Félix Guattari qui, pour rendre compte de ce fameux «entre-deux" dont ils reconnaissent l'existence primordiale tout autant que Sibony et bien avant lui, recourent non au mythe d'une origine partagée, perdue ou redonnée, morcelée ou totalisante, mais à l'affirmation de la seule réalité matérielle et intensive du devenir qui "ne produit pas autre chose que lui-même», n'a pas de sujet, ni de termes distincts de lui-même, n'est pas une évolution ${ }^{47}$, bref se déploie comme une force nue, sans entraves et sans amarres, sans passé et sans avenir. Ainsi, écrivent-ils,

[...] une ligne de devenir n'a ni début ni fin, ni départ ni arrivée, ni origine ni destination; et parler d'absence d'origine, ériger l'absence d'origine en origine, est un mauvais jeu de mots. Une ligne de devenir a seulement un milieu. Le milieu n'est pas une moyenne, c'est un accéléré, c'est la vitesse absolue du mouvement. Un devenir est toujours au milieu, on ne peut le prendre qu'au milieu. Un devenir, ajoutent-ils, n'est ni un ni deux, ni rapport des deux, mais entredeux, frontière ou ligne de fuite, de chute, perpendiculaire aux deux. Si le devenir est un bloc (bloc-ligne), c'est parce qu'il constitue une zone de voisinage et d'indiscernabilité, un no man's land, une relation non localisable emportant les deux points distants ou contigus, portant l'un dans le voisinage de l'autre, et le voisinage-frontière est indifférent à la contiguïté comme à la distance. ${ }^{48}$

Il me paraît évident, en ce sens, que Sibony, comme on peut le vérifier à la page 11 de son livre, a repris quasiment les mêmes termes employés ici, dans Mille Plateaux, par Deleuze et Guattari, mais en les inscrivant dans une conception du devenir absolument antinomique, puisque désormais soumis à une normalisation mythologique. Le devenir deleuzoguattarien, lui, au contraire, n'obéit à aucune règle, est dépourvu de toute référence et croît avec l'innocence sauvage, la vigueur incontrôlable du rhizome qui, à l'encontre de l'arborescence programmée et ordonnée ou hiérarchique de l'arbre, étend d'une façon anarchique et aléatoire ses tiges souterraines. Ce modèle rhizomatique du devenir dissout donc en même temps l'un et le multiple dans la mouvance d'une connexion indéfinie et hétérogène qui parcourt tous les ordres ou genres de la nature et de la culture ou de l'artifice ${ }^{49}$ en procédant des jeux non signifiants de «variation, expansion, conquête, capture, piqûre» d'un système énergétique ou, si l'on préfère, d'un réseau hybride d'intensités. 
Cette nouvelle approche est ainsi à la fois la récapitulation et le développement des quatres pistes tracées ou initiées par Simondon: elle réduit l'être à un devenir qui lui-même s'offre comme une «structure réticulaire amplifiante» d'intensités énergétiques hybrides en perpétuel dédoublement et, par conséquent, comme un système foncièrement métastable. Autrement dit, dans cette optique, l'altérité n'est en aucun cas une donnée empirique de quelque ordre qu'elle soit, objective ou subjective, physico-chimique ou psychique, formelle ou relationnelle, mais le processus même qui conditionne toute différenciation. Comme on le sait, Deleuze luimême trouve une illustration de cette idée dans le Robinson du Vendredi ou les limbes du Pacifique de Michel Tournier qui justement dépeint, dans son roman, une île déserte où s'efface progressivement la présence d'Autrui: «il faut comprendre», écrit-il dans un appendice de Logique du sens, qu'autrui n'est pas une structure parmi d'autres dans le champ de perception (au sens où, par exemple, on lui reconnaîtrait une différence de nature avec les objets). Il est la structure qui conditionne l'ensemble du champ, et le fonctionnement de cet ensemble, en rendant possible la constitution et l'application des catégories précédentes. Ce n'est pas le moi, c'est autrui comme structure qui rend la perception possible. 50

On peut donc dire qu'avec Deleuze associé plus tard avec Guattari, le concept d'altérité acquiert un statut de réalité transcendantale qui est celui du devenir lui-même en tant que condition a priori et autosuffisante de notre expérience sensorielle. Or précisément ce statut de réalité a-t-il lui-même une garantie, une légitimité? Ne peut-on l'imputer à un effet d'illusion? L'altérité n'est-elle pas plutôt réductible à un simple jeu? C'est en tout cas ce que croit pouvoir supposer Jean Baudrillard en proposant ainsi un septième et ultime modèle interprétatif, le plus radical parce que totalement nihiliste et que pour cette raison même, en m'autorisant de la qualification que ce penseur s'est attribuée à lui-même, j'appellerai paroxystique ou paroxyste ${ }^{51}$. Pour lui, en effet, «la réalité est une imposture. Sans vérification possible, le monde est une illusion fondamentale " 52 . Il n'y a plus de réel parce qu'il n'y a pas d'équivalent, pas de double, pas de représentation, pas de miroir du monde qui pourrait lui garantir son être dans un échange possible. Il en est ainsi de n'importe quel système aussi bien économique, politique, juridique, esthétique que biologique et physique dans la mesure où aucun d'entre eux ne peut justifier ses vues «à un niveau universel »: chacun est affecté, dit Baudrillard, "de l'indécidabilité croissante de ses catégories, de ses discours, de ses stratégies et de ses enjeux" ${ }^{53}$. D'une manière plus fondamentale encore, notre pensée qui tente de les appréhender, de les énoncer et de leur conférer une rationalité critique est en quelque sorte entraînée dans "un processus catastrophique», qui est celui «d'un dérèglement de toutes les règles du jeu » 54 non seulement par l'impossibilité de validation de ses couples de valeurs directrices comme le Vrai et le Faux, le Bien et le Mal, mais aussi par l'inadéquation, mieux l'absence d'ancrage du signe à tout référent, sa flottaison à l'instar des monnaies:

[...] il passe, écrit Baudrillard, dans la simulation et la spéculation pure de l'univers virtuel, celui de l'écran total, où la même incertitude plane sur le réel et sur "la réalité virtuelle», dès lors qu'ils ont disjoncté. Le réel ne prend plus force de signe et le signe ne prend plus force de sens. 55

Autrement dit, la virtualisation provoquée par l'inflation généralisée de la simulation nous emporterait inexorablement dans la turbulence d'«une machination» infernale où toute réalité se dérobe, devient illusion et par là s'anéantit dans une vaine identification, une sorte de «métastase indéfinie de l'identité», selon Baudrillard ${ }^{56}$, celle même du «cycle du Rien " 57 . Ainsi, aux yeux de ce penseur, le monde joue à se dédoubler pour se donner l'illusion d'exister par «la comédie du Virtuel» qui, sous toutes ses formes (le numérique, l'information, la computation universelle, le clonage), permet, d'une façon fantasmagorique, l'équivalence finale de toutes choses. Par conséquent, je ne pense plus le virtuel, mais «le virtuel me pense. Mon double erre au fil des réseaux, où je ne le rencontrerai jamais " 58 . En définitive, la dimension virtuelle, en totalisant et monopolisant aujourd'hui tout le réel, évacue toute alternative 
imaginaire, exclut toute altérité radicale et le condamne à se dévorer lui-même ${ }^{59}$ : elle nous fait accéder du même coup à «un stade paroxystique», c'est-à-dire, selon l'étymologie grecque du mot (paroxyton), situé au moment avant-dernier, juste avant la fin où il n'y a plus rien à dire et tolérant seulement la parodie comme dernière lueur de la réalité avant de disparaître ${ }^{60}$.

Avec Baudrillard, comme on le voit, nous atteignons l'approche extrême, semble-t-il, de l'altérité puisqu'à l'inverse de tous les modèles précédents, celleci non seulement perd toute référence à une réalité, être ou devenir, intentionnalité ou structure, archétype originel ou système métastable et rhizomatique, mais dissout cette réalité même dans une sorte de simulation galopante et indéfinie comme processus de virtualisation généralisée, en d'autres termes, de nivellement identitaire par équivalence illimitée. Or si une telle perspective offre, à mes yeux, l'intérêt majeur de pointer et souligner la nature et la cause du dédoublement qui affecte la totalité des apparences et les rend illusoires et instables, à savoir le fameux mécanisme de simulation, elle semble, en revanche, en proposer une interprétation triplement discutable: d'une part, par la rhétorique qui la véhicule; d'autre part, par la définition même de ce mécanisme et du virtuel qu'il est censé produire; enfin par la justification qu'elle en donne.

En effet, on ne peut pas ne pas s'étonner, tout d'abord, du statut ambivalent du discours même par lequel Baudrillard prétend et tente de nous faire connaître sa pensée: s'il consent à l'énoncer, n'est-ce pas parce qu'il croit implicitement à son pouvoir de signification et conjointement à la possibilité de nous y faire adhérer alors que, par ailleurs, il affirme précisément destituer le signe de toute légitimité, puisque sans référent et inéchangeable, et proclame à la fois le caractère illusoire de toute valeur de vérité et l'inanité de toute argumentation logique, de toute démarche rationnelle visant à convaincre l'interlocuteur éventuel? Si tout est frappé a priori d'incertitude et devient indécidable, au nom de quoi devrais-je accepter de faire crédit à un discours péremptoire qui assène des aphorismes aussi catégoriques que des oracles prophétiques? Sans doute, Baudrillard a-t-il toujours la ressource d'invoquer précisément «le cycle du Rien» dans lequel nous évoluons, pensons et parlons et qui évacue $a$ priori la contradiction et l'irrationnel et où «tout y est paradoxal» ${ }^{61}$. Mais une position aussi inexpugnable ne condamne-t-elle pas le penseur sinon à la clôture autistique, du moins, en tout cas, à l'évasion dans le cri ou à la seule issue du rêve ou du délire poétique et plus généralement de l'acte artistique, ce qui, d'ailleurs, peut ouvrir, selon moi, de vastes et radieux horizons? Son choix actuel et, semble-t-il, définitif de se consacrer exclusivement à la photographie me paraît indiquer cette prise de conscience:

[...] quand le discours arrive à une limite, avoue-t-il, il doit

s'arrêter ou se métamorphoser. L'image-photo est pour moi la

plus belle métamorphose du discours arrivé à ses confins théoriques. 62

Mais même si nous acceptons la légitimité et l'opportunité d'un tel parti pris, il reste que Baudrillard se croit autorisé à nous proposer une analyse pour étayer son propos: celle du mécanisme de simulation comme processus de virtualisation. D'où ma deuxième réserve ou interrogation: dans quelle mesure une telle analyse permet-elle de rendre compte de ce phénomène ou seulement même de l'éclairer? Tout le raisonnement de Baudrillard, en effet, repose sur un triple postulat connexe, à savoir: d'une part, toute valeur, et en tout premier lieu celle de vérité, est attestée par la possibilité d'un échange avec une réalité censée en être le référent; d'autre part, cet échange implique sa réduction à une équivalence; enfin, une telle équivalence généralisée n'est elle-même rendue possible que par l'usage d'un substitut à tout référent réel dont il constitue la négation même et offre l'illusion; substitut qu'il appelle «le virtuel» et qui est donc le résultat du processus dit de simulation comme «réversion et mise à mort de toute référence", à l'encontre même de la représentation, du reflet et du masque. Or ce triple postulat est, à mes yeux, sujet à caution pour trois raisons essentielles:

$1^{\circ}$ le rapport à la valeur non seulement n'est pas homogène et universalisable, mais il est chaque fois l'effet d'un processus singulier et unique d'élaboration $d u$ 
référent qui n'a rien à voir avec celui d'échange qui n'a lieu, lui, qu'avec des choses déjà données ou existantes. De plus, il présuppose une intentionnalité qui est totalement étrangère à celle d'une référenciation axiologique, qu'elle soit logique, éthique ou esthétique dans la mesure où celle-ci implique le maintien indispensable à la fois d'une distance et d'une dénivellation ou subordination qui la rendent précaire. $2^{\circ}$ Cet échange ne peut pas se réduire au seul principe d'équivalence puisque, d'une part, les valeurs de la chose et du référent sont d'ordres différents; et, d'autre part, que leur égalité prétendue suppose l'intervention d'une estimation qui demande chaque fois à être cautionnée.

$3^{\circ}$ Enfin et surtout le processus de simulation ne se confond en aucune façon avec la négation ou l'annulation de la réalité censée antécédente du référent, mais, comme j'ai déjà eu l'occasion de le montrer ailleurs, présuppose, au contraire, la manifestation, l'expansion et l'extension de celle-ci, en un mot, l'affirmation de son dynamisme: le virtuel en tant que simulacre n'est pas un double fou ou flottant, une fiction vide et autosuffisante, en dérive et inflation permanente, pour accréditer une équivalence illusoire, mais, selon moi, l'effet positif d'une projection immanente à notre corporéité sensori-motrice dont, bien loin d'en être le substitut, il constitue une sorte de dilatation ou d'accroissement stimulant et aléatoire. En ce sens, contrairement à Baudrillard, je dirai que le virtuel n'évacue pas "l'alternative imaginaire», mais la propulse ou la promeut dans un jeu constant.

D'où ma troisième réserve ou question concernant précisément l'illustration et surtout la justification de l'hégémonie fatale du processus de virtualisation: selon Baudrillard, elle découle inexorablement de l'essor vertigineux et incontrôlable des hautes technologies qui imposent la mise en place d'un double artificiel du monde permettant de s'y substituer, à la manière d'une écriture automatique fonctionnant en son absence et qui devient ainsi plus réelle et plus vraie que lui comme l'attestent, entre autres, par exemple, l'informatique, la robotique, le clonage. Mais là réside, à mes yeux, le malentendu: le virtuel ne se confond pas avec l'artificiel. S'il a indéniablement une réalité, ce n'est pas celle d'un substitut matériel et spatial du monde, destiné à le supplanter, mais bien plutôt celle de l'expansion, de la dilatation temporelle immanente au travail sensoriel lui-même qui, en tant que projection, ne cesse par là de se différencier, bref de devenir autre. Bien plus, la virtualité que Baudrillard prête aux artefacts technologiques n'est pas celle qu'il croit: l'image numérique n'est pas un double abusif, trompeur et autonome qui tend à produire l'illusion d'un réel référent, mais un dispositif matériel commode non seulement pour le maîtriser, mais aussi jouer avec son devenir, ses potentialités, en un mot décupler ou démultiplier notre pouvoir sur l'environnement. Autrement dit, Baudrillard, selon moi, se méprend doublement: d'une part, par sa définition philosophique de la nature et de la finalité de la simulation comme mécanisme de virtualisation; d'autre part, par son interprétation sociologique du processus technologique censé le mettre en œuvre.

Bref, si sa reconnaissance de l'opération simulatrice au sein de notre approche de l'environnement me paraît pertinente et prometteuse, il me paraît, en revanche, la dénaturer et en travestir le sens : sa radicalité est donc inadéquate et inopportune.

En fait, la raison de cette double méprise réside dans la problématique même de l'altérité qu'elle présuppose. En effet, Baudrillard appréhende cette altérité dans une prétendue «réalité» générale et constituée du monde à laquelle nous adhérerions ou croirions spontanément, qui fonderait par là même nos certitudes et qu'il convient, au contraire, selon lui, de destituer et récuser en dénonçant son «imposture» radicale ou mieux en dévoilant «la machination du Rien » qui la hante et la travaille. Mais précisément ces concepts de «réalité» et de «rien" n'ont, à mes yeux, aucune validité épistémologique: ce sont des constructions spéculatives secondaires à visée idéologique et polémique, des «idéologèmes», dirait J. Kristeva, pourvus d'une simple fonction rhétorique. Ce qui existe effectivement et concrètement, en revanche, c'est le processus sensori-moteur de la corporéité qui permet de découvrir des phénomènes 
soi-disant réels, de les énoncer, de les connaître, d'en vérifier l'authenticité et, par conséquent, de les constituer comme tels. Il faut donc, selon moi, déplacer la problématique de l'altérité en amont, au niveau et au sein de la perception même qui la fait apparaître. C'est en tout cas ce que je crois proposer en terminant à titre d'hypothèse prospective, pour permettre à la fois un accomplissement optimal, une plus fine compréhension et surtout un développement plus créatif de l'acte de danser.

En effet, comme j'ai déjà eu l'occasion de le montrer ailleurs ${ }^{63}$, notre sensorialité, par laquelle nous opérons une différenciation avec le monde physique, vivant, humain et, en premier lieu, au sein de notre propre corporéité, fonctionne comme un système chiasmatique généralisé et plus particulièrement grâce à quatre chiasmes principaux: l'un intrasensoriel parce qu'immanent à l'organe lui-même qui est simultanément actif et passif; un deuxième intersensoriel comme jeu de correspondances entre les impressions des divers organes; un troisième dit parasensoriel qui articule chaque sensation avec l'émission phonique et verbale; et enfin un quatrième intercorporel qui constitue la trame des interférences croisées de deux corporéités distinctes. Quatre chiasmes, par conséquent, qui forment une caisse de résonances hybrides ou une chambre polyvalente d'échos par un mécanisme permanent de projection par lequel chaque impression sensorielle non seulement se dédouble, mais alimente et charge cet analogon virtuel des microdifférences résultant de l'entrelacs subtil avec toutes les autres. Autrement dit, toute sensation s'accomplit par la production de simulacres qui, bien loin d'en être le simple équivalent, ne cessent de l'amplifier, de la moduler, de la déstabiliser. Bref, le travail de simulation immanent à la corporéité sensorielle est non celui d'un banal clonage, mais celui d'une dynamique de création fictionnaire qui est l'imaginaire même et qui opère conjointement et en permanence dans l'acte d'énonciation orale et scripturaire. Ainsi, de même que l'énonciateur ne peut affirmer et attester son identité qu'en se projetant dans l'altérité scénique d'une parole prononcée ou d'une écriture tracée, de même et plus radicalement les sensations qu'il éprouve simultanément, et qui sous-tendent et suscitent son discours, ne surgissent et ne peuvent être identifiées que par l'altérité du processus fictionnaire qui les habite. Celle-ci est donc bien la matrice originaire, incontournable, nécessaire et fondatrice de toute notre corporéité et de sa nostalgie identitaire. En ce sens, toutes les altérités ultérieures, aussi bien culturelles et artificielles que naturelles et primitives ou soit-disant innées que nous avons inventoriées plus haut, ne sont que différentes manières de rejouer cette ruse primordiale et cachée de notre imaginaire sensoriel en essayant toujours davantage de nous sécuriser par la quête et le mirage d'une identité factice.

Or il est bien évident que c'est dans l'acte de danser qu'on peut le mieux discerner les manifestations de cette altérité originaire, même si beaucoup de créations chorégraphiques contemporaines ont malheureusement tendance à l'occulter ou travestir par le recours à des artifices propres à accréditer l'idée d'une danse comme communication œcuménique d'identités diverses. En effet, on peut le vérifier au niveau des quatre principaux paramètres qui caractérisent, selon moi, la corporéité dansante à la fois dans l'expérience vécue de l'interprète et dans sa visibilité spectaculaire, à savoir, je le rappelle: une dynamique de métamorphose indéfinie, un jeu aléatoire de tissage et détissage de la temporalité, un défi ou dialogue obstiné avec la force gravitaire et une pulsion autoaffective. Quatre paramètres, comme on peut le constater, qui présupposent tous la mise en œuvre et la manifestation d'une altérité continuée, aussi bien spatiale que temporelle, active qu'expressive, en quelque sorte, celle d'une mutation ininterrompue. Or précisément cette altérité prend sa source dans celle qui régit la production du mouvement, c'est-à-dire plus particulièrement les postures, les attitudes, les déplacements, les gestes et les expressions. Ainsi, en ce qui concerne la posture et l'attitude, Hubert Godard a fort bien montré qu'elles procèdent fondamentalement de la tension provoquée par la gestion des muscles gravitaires, autrement dit du mode de résolution du conflit avec le poids permettant la station debout ou l'équilibre, ce 
qu'Hubert Godard appelle "le pré-mouvement»: toute posture et a fortiori toute attitude qui la module sont, en ce sens, l'épiphanie d'un drame neurophysiologique, d'une altérité profonde qui tente de se travestir dans le simulacre d'une identité éphémère, mais, comme telle, porteuse d'une charge émotionnelle inévitable. Pour l'illustrer, Hubert Godard cite opportunément le merveilleux solo de Trisha Brown, If you could'nt see me créé en 1994, où elle ne donne à voir que son dos nu avec ses multiples jeux musculaires qui constituent en quelque sorte la mise en scène des tensions exercées avec la colonne vertébrale qui assurent son équilibre et l'apparition de tous les autres mouvements ${ }^{64}$.

Toutefois l'apparition de ces mouvements, déplacements et gestes nécessite aussi, par-delà la gestion temporaire de ce conflit postural, la mise en œuvre d'une seconde dimension de l'altérité originaire de notre corporéité: celle qui sous-tend et anime le processus sensoriel qu'ils véhiculent. En effet, aussi bien les mouvements transitifs de déplacement dans l'espace et conjointement d'action utilitaire que les gestes réflexifs et spontanés visent avant tout à modifier notre appréhension sensorielle des choses ou plus généralement de notre environnement. Or cette appréhension implique toujours, comme nous l'avons montré, une simulation, c'est-à-dire une projection immanente de fictions qui confèrent à chaque objet perçu un dessin et une charge imaginaire dont le danseur ou la danseuse est le meilleur réceptacle et en même temps producteur ou agent. L'exemple le plus évident et le plus significatif de ce phénomène se trouve dans les solos de Dominique Dupuy qui, comme je l'ai analysé dans une récente communication ${ }^{65}$, tente d'opérer la transmutation à la fois poétique et chorégraphique des choses. Pour cela, sa corporéité vise à restituer et rendre visible par ellemême les diverses qualités sensibles qui constituent chaque chose et que ses différents organes sensoriels enregistrent et mêlent: forme, volume, couleur, texture, poids, fonctionnalité, signification. Ainsi choisit-il, par exemple, de mouler et plier son corps en écho à la convexité ou à la sphéricité des ballons dont il s'empare et avec lesquels il joue, ou bien encore de convertir la tactilité de la résistance d'un matériau dans les mouvements violents, frénétiques et désordonnés de la tête et des jambes qui laissent éclater simultanément l'intensité du désir qui essaie de l'investir. La corporéité du danseur devient alors l'effigie de l'altérité insurmontable du travail de simulation qu'effectue sa sensorialité.

Mais, comme on le sait, ce travail déborde la seule sensorialité inhérente à nos actes et nos gestes: il régit la totalité de notre corporéité expressive. Comme j'ai essayé de le montrer dans ma thèse ${ }^{66}$, la catégorie d'expressivité corporelle implique non seulement la production énergétique d'une dynamique de rejet, mais aussi et surtout un mécanisme de différenciation immanente suscité par une pulsion autoaffective, qui tend à faire ressusciter en soi la présence de l'objet de désir et ainsi à s'accorder une satisfaction anticipée et fictive. Autrement dit, l'expressivité ne fonctionne que par l'altérité radicale d'un processus fictionnaire dont la voix constitue l'archétype: toute expression corporelle, visible et audible, est donc essentiellement et de part en part «transvocalisation». Or, selon moi, Mary Wigman, d'une certaine façon, l'avait fort bien pressenti quand elle déclarait que tous ses solos se déroulaient avec «un partenaire invisible» et imaginaire qui polarisait et investissait l'expression de son regard, de sa face, de la tension et de la direction de ses bras, la position et l'ouverture de ses mains, l'extension de ses doigts, les déplacements et les flexions de ses jambes et fondamentalement le choix et les modalités de ses appuis sur le sol. Mais hélas ce duo imaginaire de l'expression dansée ne représentait pour elle qu'une «intrigue » 67 , où seule l'intéressait la puissance dramatique ou conflictuelle qu'elle dégageait.

C'est pourquoi Cunningham a, avec raison, récusé cette vision expressionniste de la corporéité dansante pour dévoiler et promouvoir, en revanche, l'expressivité immanente au mouvement, «au delà de toute intention " ${ }^{6}$. Même s'il ne justifie pas théoriquement la pertinence de son propos (ce qui est, à ses yeux, superflu), il me paraît deviner la force de l'altérité intrinsèque de la corporéité du danseur sans pour autant néanmoins faire affleurer sa dimension autoaffective. Trisha Brown, en revanche, semble, selon 
moi, l'avoir fort clairement perçue et je dirais volontiers avec Hubert Godard que, chez elle, la corporéité ne cesse de se nourrir de son propre mouvement et qu'elle laisse sa sensibilité être affectée par lui:

[...] le danseur, chez Trisha Brown, écrit H. Godard, n'est pas tant fidèle à l'espace qui l'entoure qu'attentif à une dynamique particulière du mouvement qui nécessite une écoute et un ressenti de la phrase vécue dans la plus infime trace de son origine: dans le prémouvement lui-même. 69

Or c'est cette écoute autoaffective, cette réflexivité sensori-motrice avec son altérité implicite qui confère à chaque expression sa singularité unique, mais qui aussi, notons-le, risque toujours simultanément et malheureusement, comme on le voit chez certains jeunes chorégraphes contemporains, de se niveler et sombrer dans l'identité d'un banal et fastidieux narcissisme.

Ainsi, et ce sera là ma conclusion, il y a une altérité originaire et fondatrice de notre corporéité, mais qui reste foncièrement ambivalente: si elle constitue la matrice primordiale de toute création artistique, elle ne peut l'être que par l'irruption simultanée, menaçante et sournoisement pernicieuse du désir d'identité qui la hante.

\section{N O TES}

1. V. Descombes, Le Même et l'Autre, Paris, Minuit, 1993, p. 13.

2. F. Jacob, La Souris, la mouche et l'homme, Paris, O. Jacob, 1997, p. 163.

3. Ibid., p. 153.

4. L'Identité (Séminaire dirigé par C. Lévi-Strauss), Paris, Grasset, 1977.

5. T. Todorov, Nous et les Autres. La réflexion française sur la diversité humaine, Paris, Éd. du Seuil, 1989, p. 421-437.

6. J.Kristeva, Étrangers à nous-mêmes, Paris, Fayard, 1989.

7. V. Descombes, op. cit.

8. E. Levinas, Autrement qu'être ou au-delà de l'essence, Dordrecht, Kluwer Academic Publishers, 1988; Le Temps et l'Autre, Paris, P.U.F., 1993.

9. J. Baudrillard, L'Autre par lui-même. Habilitation, Paris, Galilée, 1987. 10. J. Baudrillard, L'Échange impossible, Paris, Galilée, 1999.

11. M.Merleau-Ponty, Phénoménologie de la perception, Paris, Gallimard, 1945, p. 469

12. L. Brunel, G. Delahaye et B. Mangolte, Trisha Brown, Paris, Éd. Bougé, 1987, p. 44.

13. A. Lepecki, «Par le biais de la présence: la composition dans l'avantgarde postbauschienne", Nouvelles de Danse 36/37: La Composition, 1998, p. 193.

14. E. Husserl, Méditations cartésiennes, Paris, Librairie Vrin, 1969, p. 78.

15. M. Merleau-Ponty, op. cit., $2^{\text {e }}$ partie, chap. IV, p. 401 et 407.

16. E. Levinas, op. cit., 1988.

17. E. Husserl, op. cit., p. 133.

18. J. Derrida, "Violence et métaphysique», 2e partie, Revue de Métaphysique et de Morale, n 4, octobre-décembre 1964, p. 450 et plus généralement p. 438 ss.

19. V. Descombes, op.cit., p. 127.
20. C. Lévi-Strauss, op.cit., p. 332.

21. M. Merleau-Ponty, Signes, Paris, Gallimard, 1960, p. 154

22. C. Lévi-Strauss, Tristes Tropiques, Paris, Plon, 1955, p. 50.

23. V. Descombes, op.cit., p. 129.

24. Ibid., p. 123. Pour une étude plus approfondie du structuralisme, je renvoie le lecteur à l'ouvrage collectif intitulé Qu'est-ce que le structuralisme?, publié par les Éd. du Seuil en 1968 par O. Ducrot, T. Todorov, D. Sperber, M. Safouan et F. Wahl.

25. G. Simondon, L'Individu et sa genèse physico-biologique, Paris, P.U.F., 1964.

26. Ibid., p. 17.

27. Ibid., p.5.

28. Ibid., p.6.

29. Ibid., p. 9.

30. Ibid., p. 10.

31. Ibid., p. 11.

32. Ibid., p. 12 et 15

33. Ibid., p. 15.

34. Ibid., p. 22.

35. Ibid., p. 16.

36. Ibid., p. 18.

37. Ibid., p. 20-22.

38. G. Devereux, Ethnopsychanalyse complémentariste, Paris, Flammarion, 1985; D. Sibony, Entre-deux. L'origine en partage, Paris, Seuil, 1991.

39. T. Nathan, La Folie des autres. Traité d'ethnopsychiatrie clinique, Paris, Dunod, 1986 et Psychanalyse païenne. Essais ethnopsychanalytiques, Paris, O. Jacob, 1995.

40. D. Sibony, op. cit., p. 10-11.

41. Ibid., p. 16.

42. Ibid., p. 352.

43. Ibid., p. 16-17.

44. Ibid., p. 340.

45. Ibid., p. 343-347.

46. Ibid., p. 343-344

47. G. Deleuze et F. Guattari, Mille Plateaux, Paris, Minuit, 1980, p. 291-292.

48. Ibid., p. 360.

49. Ibid., p. 32.

50. G. Deleuze, Logique du sens, Paris, Minuit, 1969, p. 358.

51. J. Baudrillard, Le Paroxyste indifférent. Entretiens avec Philippe Petit, Paris, Grasset, 1997.

52. J. Baudrillard, L'Échange impossible, op.cit., p. 11.

53. Ibid., p. 12.

54. Ibid., p. 29.

55. Ibid., p. 13-14.

56. Ibid., p. 72 .

57. Ibid., p. 18.

58. Ibid., p. 24-25.

59. J. Baudrillard, Le Paroxyste indifférent, op. cit., p. 80, 95.

60. J. Baudrillard, L'Échange impossible, op. cit., p. 35.

61. Ibid., p. 17,18 et 29.

62. Cf. "Le corps, un échange impossible?», Entretien de J. Baudrillard, Revue Prétentaine, "Numéro spécial Corps", mars 2000 (Université P. Valéry Montpellier III), p. 275.

63. M. Bernard, «Sens et fiction ou les effets étranges de trois chiasmes sensoriels", Nouvelles de Danse, ${ }^{\circ} 17$, octobre 1993, p. 56-64.

64. H. Godard, «Le geste et sa perception", dans La Danse au XXe siècle d'I. Ginot et M. Michel, Paris, Éd. Bordas, 1995, p.224 et 228.

65. M. Bernard, «Dominique Dupuy, danseur alchimiste ou du pouvoir poétique de transmutation chorégraphique des objets ", à paraître dans mon prochain ouvrage: De la création chorégraphique.

66. M. Bernard, L'Epressivité du corps. Recherches sur les fondements de la théâtralité, 1re éd. J.P. Delarge 1976; 2e éd. Chiron, 1986, chap. V et VI. 67. M. Wigman, Le Langage de la danse, Paris, Papiers, 1986, p. 22. 68. M. Cunningham, Le Danseur et la Danse. Entretiens avec J. Lesschaeve, Paris, Belfond, 1980, p. 129.

69. H. Godard, op. cit., p. 228. 